\title{
Research on Energy-Saving Experimental of Critical Dehumidification of Combined Drying by Dehumidification Wheel and Heat Pump
}

\author{
Jiaoling Wang $(\mathbb{D}$, Weidong Song, Chengqian Jin $(\mathbb{D}$, Tianhang Ding, Mingyou Wang, \\ and Jinji $\mathrm{Wu}$
}

Nanjing Institute of Agricultural Mechanization, Ministry of Agriculture and Rural Affairs, Nanjing, China

Correspondence should be addressed to Chengqian Jin; 412114402@qq.com

Received 3 December 2020; Revised 18 February 2021; Accepted 29 April 2021; Published 12 June 2021

Academic Editor: Walid Elfalleh

Copyright ( 2021 Jiaoling Wang et al. This is an open access article distributed under the Creative Commons Attribution License, which permits unrestricted use, distribution, and reproduction in any medium, provided the original work is properly cited.

\begin{abstract}
In order to further decrease the energy consumption of desiccant wheel dehumidification, the drying medium circulation characteristics of a system combining heat pump drying with desiccant wheel dehumidification were investigated. Moreover, the critical dehumidification conversion mechanism was studied. The analysis of the heat pump hot air circulation system demonstrated that the heat pump system has the best dehumidification efficiency. Through the analysis of the system combining heat pump drying with desiccant wheel dehumidification, the critical conversion point was determined. The critical dehumidification mechanism was further verified using an online temperature and humidity measurement system. To investigate the effect of the critical point on energy consumption and drying quality and develop a drying model, response surface experiments were performed based on the effects of regeneration temperature, drying temperature, and conversion point relative humidity on rehydration, color difference, and specific moisture extraction rate (SMER). The optimal conversion point humidity was determined to be about $46 \% \mathrm{RH}$, which was slightly different from the test optimization value of $45.6 \% \mathrm{RH}$. In addition, comprehensive optimization and experimental verification of the influencing factors were conducted. The results demonstrated that the $R^{2}$ values of the three models were greater than 0.98 , and the experimental factors had a significant effect on drying quality and energy consumption. When the regeneration temperature was $96^{\circ} \mathrm{C}$, the drying temperature was $53^{\circ} \mathrm{C}$, the relative humidity of the conversion point was $46 \%$, the color difference was 46.3 , the rehydration ratio was 5.75 , and the $S M E R$ was $1.62 \mathrm{~kg} / \mathrm{kW} \cdot \mathrm{h}$.
\end{abstract}

\section{Introduction}

Drying agricultural products is an important means for reducing losses and facilitating safe storage. Moist agricultural products are prone to mold growth and can produce toxins, such as aflatoxin, which are harmful to human health. Common heat transfer-based drying methods include radiation, conduction, and convection drying [1]. Among them, convection drying is the most widely used, due to its simple equipment and wide application range $[2,3]$. The main adjustable parameters related to convection drying are the air temperature, air speed, and humidity of the drying medium. In each drying stage, the temperature has an upper limit. Exceeding this upper limit may damage the quality of the agricultural products $[4,5]$. The adjustment of the air volume should not be too large; otherwise, it is not conducive to the full heat exchange between materials. In most stages of drying, the humidity of the drying medium is not limited $[6,7]$. According to the second law of Fick diffusion, low humidity can increase the concentration gradient between the surface of the material and the drying medium, increase the drying rate, and achieve low-temperature drying $[8,9]$. Consequently, humidity is an ideal adjustment parameter.

Common dehumidification methods include condensation, adsorption, osmosis, and combined dehumidification. Desiccant wheel dehumidification is a common type of adsorption dehumidification, which has the advantages of 
independent and deep dehumidification [10]. However, to provide continuous drying conditions, it requires continuous regeneration with a small amount of dehumidification. Heat pump drying is a common type of condensation dehumidification with high coefficient of performance [11]. However, the dehumidification dew point is limited. Therefore, by combining heat pump dehumidification and desiccant wheel dehumidification, the advantages of the two methods can be fully integrated, providing efficient and rapid dehumidification.

At present, while heat pump drying has found relatively mature applications in the field of agricultural drying [12], the equipment related to desiccant wheel dehumidification is relatively simple. The main problems are due to unreasonable design and high energy consumption, which is associated with the regeneration technology and return air circulation. Nowadays, the hot air cycle of desiccant wheel dehumidification can be in the form of different configurations, which mainly include the Pennington cycle, Dunkle cycle, and SENS cycle in the heating, ventilation, and air conditioning (HVAC) field [13]. The traditional convection drying cycle using box-type dryers can be open, semiopen, and closed [14], all of which enable rigid drying. In addition, the circulation method with bypass rate is not applicable, since it does not meet the low dehumidification point requirement of desiccant wheel dehumidification.

To sum up, scholars have paid more attention to the HVAC of desiccant wheel dehumidification [15]. Nevertheless, the cycle of the traditional convection drying method using box-type dryers is either an open, semiopen, or closed rigid drying cycle, which cannot effectively utilize the constantly changing fresh or return air [16]. Either cycle with bypass rate can be mixed at a certain proportion of return air to achieve energy-saving drying [17]; however, it does not meet the low dew point dehumidification requirements of desiccant wheel dehumidification. In this paper, the dehumidification characteristics of heat pump drying are analyzed. Based on the drying requirements of agricultural products and the characteristics of the combined desiccant wheel dehumidification and heat pump drying system, a flexible dehumidification method for a constantly changing air return and fresh air system is proposed, aiming to achieve energy-saving drying of agricultural products.

\section{Materials and Methods}

\subsection{Drying Process Analysis}

2.1.1. Air Circulation of the Heat Pump Drying System. In the heat pump drying process, the air is cooled and dehumidified by the evaporator and then heated by the condenser to the required temperature before entering the drying chamber [18]. After having absorbed the moisture of the dried product in the drying chamber, the air returns to the evaporator, which absorbs part of the latent and sensible heat and dehumidifies it to complete the cycle [19].

During the heat pump drying process, the air circulation state changes, as shown in Figure 1, where point 1 represents the state of the dry hot air that enters the drying chamber. In

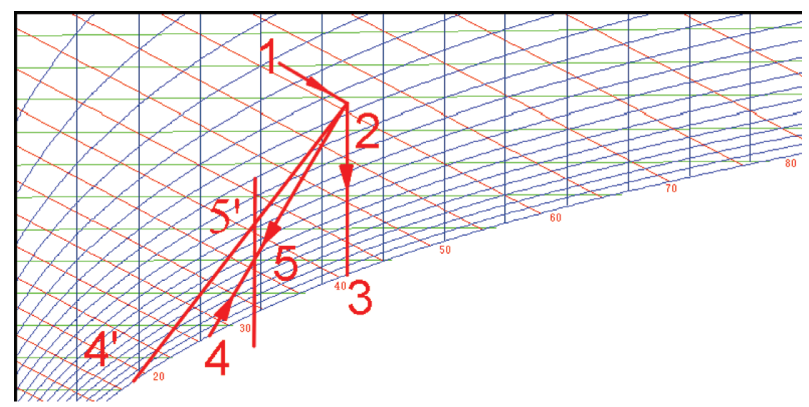

FIgURE 1: Heat pump dehumidification process.

the drying chamber, the air comes into contact with the drying material and heats it. As a result, the moisture of the material evaporates into the air, the temperature at the outlet of the drying chamber decreases, and the relative humidity of the air increases. The process taking place in the drying chamber can be considered as an isenthalpic process [20]; that is, the air changes along the isenthalpic line in the drying chamber.

The most important part of dry air circulation during heat pump drying is the dehumidification process taking place in the evaporator [21]. In Figure 1, point 2 represents the air leaving the drying tank and entering into the evaporator. It can be considered that, after saturation, part of the air is equally wet cooled to point 3 , along the saturation line of dehumidification to point 4 , and then mixed with another part of the air at point 5 . Subsequently, wet heating is conducted until the temperature required to dry the material in the drying tank is reached.

It is assumed that, after cooling, part of the air that enters the evaporator is dehumidified along the saturation line of the 2-3-4 process. The other part remains unchanged, and then, the two parts are mixed along the 2-4 line with point 5 .

The mass flow ratio of the dehumidified air to the air passing through the evaporator is expressed as PT, the value of which can effectively indicate the effect of evaporation and dehumidification. It can be expressed by the state parameter of air as [22]

$$
\mathrm{PT}=\frac{d_{2}-d_{5}}{d_{2}-d_{4}},
$$

where $d_{2}$ and $d_{5}$ are the moisture content at points 2 and 5 , respectively, and $h_{2}$ and $h_{5}$ are the enthalpy at points 2 and 5 , respectively.

The value of PT is determined by many factors. When the structure of the evaporator is standard, it is only related to the velocity and physical properties of air. It can be determined by an empirical equation (equation (2)), which is used in several engineering applications.

$$
X_{d}=\frac{d_{2}-d_{5}}{h_{2}-h_{5}},
$$

where $X_{d}$ is the dehumidification rate of the drying cycle, which is defined as the ratio of the dehumidification amount of the air passing through the evaporator to the heat released by the air. 
According to equation (2), the dehumidification rate of the drying cycle is not affected by the state at point 5 , but it is only related to that at points 2 and 4 . In general, the smaller the slope of line 2-4, the larger the value of $X_{d}$ and the more effective the dehumidification of the system. When line 2-4 is tangent to the saturated air line, $X_{d}$ reaches its maximum value, and the tangent point $t_{4}$, represents the optimal dehumidification temperature of the system.

Based on the above analysis, it can be concluded that after the state of point 2 has been determined, the dehumidification rate of the drying cycle is related to the dehumidification temperature $t_{4}$, which is the highest at $t_{4}$ '. Nevertheless, in the heat pump drying cycle, when the condensation temperature is constant, the heating capacity of the system will decrease with the decrease of the evaporation temperature, while the refrigeration coefficient COP of the system will increase. Therefore, there is an optimal dehumidification rate, which provides a reasonable match between the dehumidification effect of the entire heat pump drying system and the refrigeration coefficient COP of the system. The optimal dehumidification rate is defined as follows:

$$
\begin{aligned}
X_{e} & =\operatorname{COP} \cdot X_{d}=\frac{h_{2}-h_{5}}{W} \cdot \frac{d_{2}-d_{5}}{h_{2}-h_{5}}=\frac{d-d_{5}}{W}, \\
\operatorname{COP} & =\frac{q_{2}}{W_{0}}=\frac{h_{2}-h_{5}}{W},
\end{aligned}
$$

where $q_{2}$ is the heat released by low-temperature heat sources $(J) ; W_{0}$ is the work done by the external environment on the low-temperature reverse Cano machine $(J)$; and $W$ is the work done by the compressor $(J)$.

That is, the dehumidification rate is equal to the ratio of the dehumidification amount of the heat pump drying system to the cycle work. To maximize the dehumidification rate of the system, the optimal evaporation temperature in the heat pump drying system needs to be determined. The dehumidification rate of the system is not only related to points 2 and 4 but also related to the condensation temperature of the heat pump drying cycle.

If the entire amount of air that passes through the evaporator participates in dehumidification, under the condition of a certain cooling capacity of the heat pump dehumidifier, and if point 2 is distant from the saturation line and line 2-3 is longer, the response of line 3-4 will become shorter and the wet air displacement will decrease. When the inlet temperature of the evaporator is high and the humidity is low, point 4 may coincide with point 3 . At this point, only cooling is required without dehumidification.

If the wet air leaves the evaporator, the temperature reaches the dew point temperature $t_{\mathrm{d}}$ marginally, and the moisture content of the air flowing through the evaporator remains unchanged $\left(d_{3}=d_{2}\right)$. At this point, the enthalpy of the air entering the evaporator is expressed as $h_{\text {ein }}$.

$$
h_{\text {ein }}=h_{3}+\Delta h \text {, }
$$

where $\Delta h$ is the difference in the enthalpy of the air flowing through the evaporator $(\mathrm{kJ} / \mathrm{kg})$.
According to the properties of wet air, when the refrigerating capacity and moisture content are constant, the wet air with an enthalpy value larger than $h_{0}$ flowing through the evaporator cannot become saturated and remove the moisture. Only when the enthalpy value $h_{2}$ is lower than $h_{\text {ein }}$, the moisture can be separated out from the evaporator. Thus, the enthalpy of the initial state that corresponds to the final state of dehumidification is defined as $h_{\text {crt }}$. Consequently, the necessary condition for the dehumidification efficiency to be larger than zero is [23]

$$
h_{\text {ein }}<h_{\text {crt }} .
$$

To conclude, there is an optimal dehumidification rate, which is related to the evaporation temperature, condensation temperature, and dehumidification air enthalpy.

\subsubsection{Air Circulation Analysis in the Combined Dehumidi-} fication System. The combined dehumidification process is shown in Figure 2, where 5-1 is the product dehumidification. From point 1 to point 2 on the saturation line, the air exits from the evaporator. Then, it gets dehumidified at point 3 , enters the wheel at point 4 , and moves to point 5 through the condenser or heater. Both material drying and desiccant wheel dehumidification are similar to isenthalpic dehumidification. Therefore, lines 5-1 and 3-4 are parallel; thus, $d_{M}-d_{5}=d_{3}-d_{4}$ and $d_{1}-d_{m}=d_{2}-d_{3}$; that is, $d_{1}-d_{5}=\left(d_{2}-d_{3}\right)+\left(d_{3}-d_{4}\right)$. This indicates that, in a closedcycle drying process, the amount of moisture removed by the heat pump and the desiccant wheel is the same as the amount of water removed by the air from the material. The condensation dehumidification heat pump has the advantage of removing high moisture content and the temperature at point 3 is relatively small. Consequently, the low air temperature at the evaporator is conducive to wheel dehumidification.

The drying of materials in the drying box corresponds to isenthalpic drying. Wheel dehumidification is also an isenthalpic process. It should be noted that the lower the dehumidification temperature, the higher the wheel dehumidification efficiency. In general, the drying temperature is set in advance, before drying. During the dehumidification stage, the enthalpy gradually decreases, and the temperature of the return air gradually increases. During drying, the air moves along the isenthalpic line in the direction of cooling. In the different cycles of the drying process, the enthalpy of the return air is reduced, and its temperature increases. At the same time, with the increase of the return air temperature, if the entire amount of return air is used, the evaporation temperature increases. Due to the increase in the unit cooling capacity and gas transmission coefficient, the corresponding cooling capacity increases as well.

In the previous section, it was presented that the dehumidification capacity of the heat pump is independent of point 5 and is determined only by points 2 and 4 . However, in the combined dehumidification system, the position of point 5 has a significant effect on the dehumidification performance of the system. The dehumidification capacity of the desiccant wheel is closely related to the temperature and 


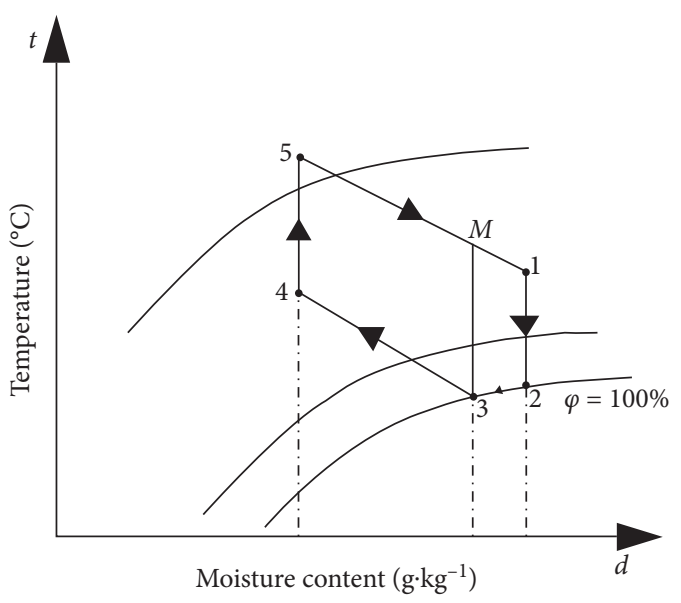

Figure 2: Combined desiccant wheel and heat pump dehumidification process.

relative humidity of the inlet air. Under the same conditions, a low inlet air temperature and high relative humidity are beneficial to dehumidification. Consequently, when point 3 coincides with point 4 , the air temperature after evaporation and dehumidification is the lowest and the relative humidity is the highest.

When the dew point of the air that enters the evaporator is lower than the evaporation temperature, the water will be separated out after condensation. When the inlet air temperature is high, the humidity is low, and the dew point temperature is higher than the evaporation temperature, the air will only be cooled after passing the evaporator without being dehumidified.

First, the situation of dehumidification after evaporation will be discussed. In order to make full use of the residual heat and air energy, the optimal conversion point of fresh and return air can be determined according to the enthalpy values of the fresh air and return air after passing through the evaporator [24].

The dotted cd line in Figure 3 shows the case when the air cools down without being dehumidified after passing through the evaporator [24]. When the air is at low temperature and has high relative humidity after leaving the evaporator, it is conducive to desiccant wheel dehumidification.

The enthalpy of the air before evaporation can be calculated by equation (6) [25].

$$
I_{0}=(1.01+1.84 d) t+2490 d,
$$

where $I_{0}$ is the enthalpy $(\mathrm{kJ} / \mathrm{kg}) ; t$ is the drying temperature $\left({ }^{\circ} \mathrm{C}\right)$; and $d$ is the moisture content $(\mathrm{g} / \mathrm{kg})$.

The refrigerating capacity of the evaporator can be calculated to determine the cycle system of the heat pump. Subsequently, the number of compressors, evaporators, and other components is determined by the heat pump system. The evaporating temperature $t_{\mathrm{e}}$ and the condensing temperature $t_{c}$ can be determined by the heat transfer temperature difference $\Delta t$. The refrigerating capacity $Q_{\mathrm{e}}$ can be determined by ARI polynomials, which have been defined by the Air Conditioning and Refrigeration Institute (ARI), and their fitting terms $[26,27]$. Therefore, the difference in the

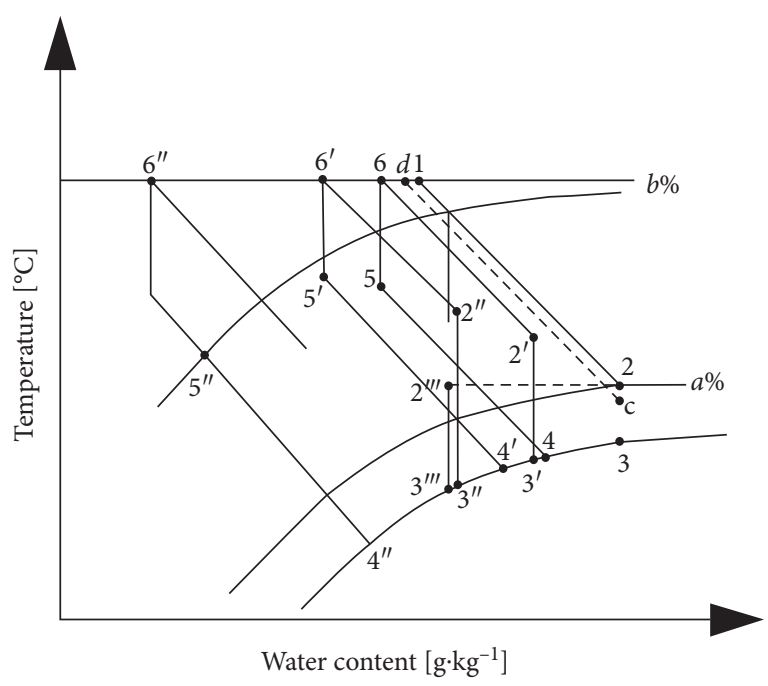

Figure 3: Critical dehumidification process.

enthalpy $\Delta I$ before and after the air passes through the evaporator is determined by equation (8):

$$
\begin{aligned}
& Q_{e}=f\left(t_{c}, t_{e}\right), \\
& \Delta I=\frac{Q_{e}}{V_{\mathrm{am}}},
\end{aligned}
$$

where $t_{\mathrm{e}}$ is the evaporation temperature $\left({ }^{\circ} \mathrm{C}\right) ; t_{\mathrm{c}}$ is the condensation temperature $\left({ }^{\circ} \mathrm{C}\right) ; Q_{\mathrm{e}}$ is the refrigerating capacity; $V_{\mathrm{am}}$ is the mass flow rate $(\mathrm{kg} / \mathrm{h})$; and $\Delta I$ is the difference in the enthalpy $(\mathrm{kJ} / \mathrm{kg})$.

The enthalpy of the air after passing through the evaporator can be determined by

$$
I_{1}=I_{0}-\Delta I,
$$

where $I_{1}$ is the enthalpy of the air at the outlet of the evaporator.

The enthalpy of the fresh air after evaporation is denoted as $I_{11}$, and that of the return air after evaporation is denoted as $I_{12}$. When $I_{12}-I_{11}<0$, drying is performed with return air; otherwise, drying is performed with fresh air [28].

2.2. Experimental Method and Equipment. As it can be seen in Figure 4, the test system consisted of a heat pump and a desiccant wheel. Temperature and humidity sensors were placed before and after the evaporator, desiccant wheel, and drying box. Moreover, the return air pipeline was equipped with a switching mechanism, which enabled the conversion of fresh air and return air. Data collection was performed by a wireless temperature and humidity tester, and the values of enthalpy and other parameters were obtained by calculation.

\subsection{Methods and Design of the Critical Dehumidification Experiment}

2.3.1. Test Objective. The aim of this experiment was to verify the energy-saving characteristics of critical 


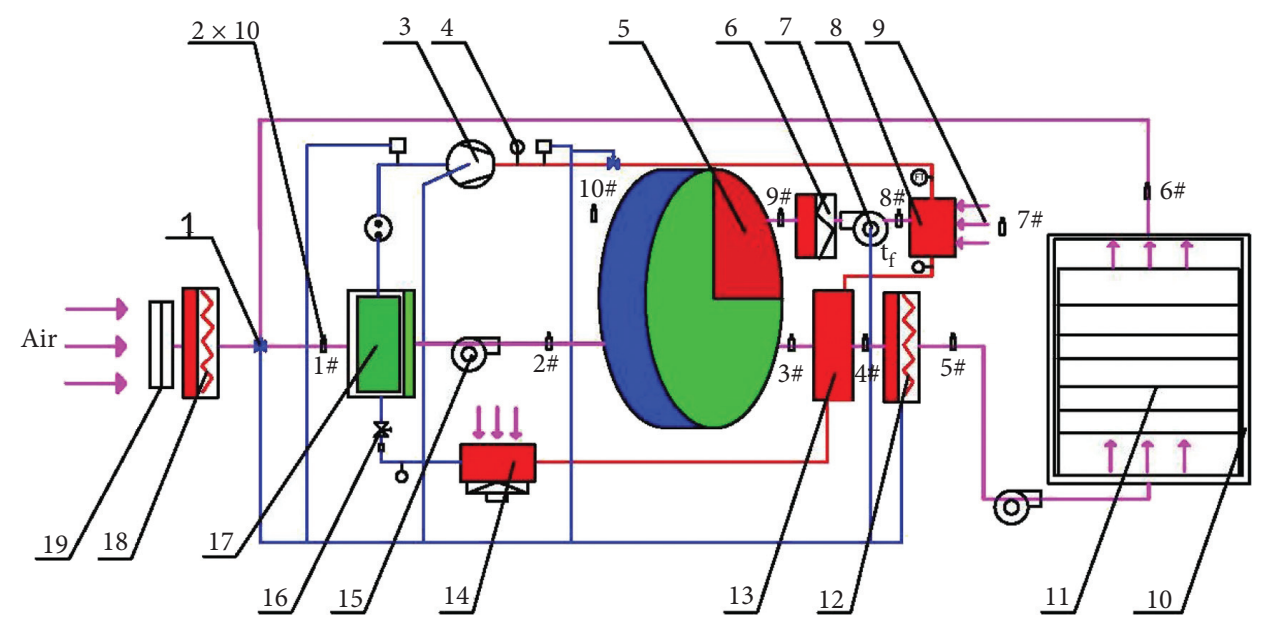

Figure 4: Experimental bench for critical dehumidification. 1: three-way valve, 2: temperature and humidity sensors, 3: compressor, 4: pressure gage, 5: desiccant wheel, 6: regenerative auxiliary heater, 7: regeneration fan, 8: regenerative condenser, 9: inlet air regeneration, 10: drying box, 11: drying frame, 12: drying auxiliary heater, 13: drying condenser, 14: waste heat condenser, 15: dehumidification fan, 16: expansion valve, 17: evaporator, 18: preheater, and 19: humidifier.

dehumidification, determine the best conversion point in the desiccant wheel dehumidification process, and develop a mathematical model of energy consumption, appearance, and other critical indicators that can provide a reference for energy-saving drying through desiccant wheel dehumidification.

\subsubsection{Materials and Instruments. Fresh Lentinus edodes} were used for the response surface experiments and Auricularia auricula for the system tests. Their moisture content was about $90 \%$ and $85 \%$, respectively. A OHAUS MB27 fast moisture tester was used to determine the moisture content. A Konica Minolta chromometer CR-10 Plus was used to measure the color differences. A Hh- 4 Thermostatic water bath pot was used to determine the rehydration ratio. A BSA224S electronic balance was used to measure the weight of Lentinus edodes. Finally, an electric meter was used to measure the power consumption of the dried Lentinus edodes.

\subsubsection{Composition Measurements}

(1) Moisture Content. Moisture content was measured with an OHAUS MB27 fast hydrometer.

(2) Rehydration Ratio. The rehydration performance of the Lentinus edodes was expressed by the rehydration ratio, which is the ratio of the Lentinus edodes mass after rehydration for a certain amount of time to that before rehydration:

$$
R_{R}=\frac{M_{f}}{M_{g}}
$$

where $R_{\mathrm{R}}$ is the rehydration ratio; $M_{\mathrm{f}}$ is the mass of the dried Lentinus edodes after rehydration ( $\mathrm{kg})$; and $M_{\mathrm{g}}$ is the mass of the Lentinus edodes before rehydration ( $\mathrm{kg})$.
During testing, a dried Lentinus edodes sample with a certain mass was weighed and put into distilled water.

(3) Color Difference Determination. The color difference was measured by Konica Colorimeter of CR-10. The color difference was reflected by the value of $\mathrm{L}$. Each sample was measured 3 times on average, and the results were averaged [29].

(4) Specific Moisture Extraction Rate. The specific moisture extraction rate (SMER) represents the amount of water that can be removed by consuming a unit of energy. In the tests, the consumed electric quantity was measured by an electricity meter, and its calculation was based on the mass of water removed in the test.

$$
\text { SMER }=\frac{M}{Q},
$$

where $Q$ is the power consumption $(\mathrm{kW} \cdot \mathrm{h})$ and $M$ is the weight of water removed by drying $(\mathrm{kg})$.

2.3.4. Response Surface Optimization Test. A response surface test was performed for a regeneration temperature $X_{1}$, drying temperature $X_{2}$, and relative humidity of the conversion point $X_{3}$, using the Box-Behnken center composite design, with the color difference $Y_{1}$, rehydration ratio $Y_{2}$, and dehumidification energy consumption $Y_{3}$ of unit energy consumption as response values. The drying time of each group was $6 \mathrm{~h}$. According to the properties of Lentinus edodes found in the related literature, their drying temperature is generally about $60^{\circ} \mathrm{C}$. In the regression and orthogonal test, the optimal drying temperature was generally greater than $50^{\circ} \mathrm{C}$, while the regeneration temperature was generally not lower than $80^{\circ} \mathrm{C}$ and not higher than $120^{\circ} \mathrm{C}$. Meanwhile, due to the isenthalpic dehumidification characteristics of the desiccant wheel, the higher level of the regeneration temperature was $105^{\circ} \mathrm{C}$. The relative humidity of the conversion points was selected based on the analysis of 
the conversion mechanism in Section 2.1.2. Based on the measurement of the initial return air enthalpy combined with the outdoor relative humidity, it was determined that the relative humidity of points $\mathrm{B}$ and $\mathrm{A}$ was $40 \%$ and $50 \%$, respectively. The test factors and levels are shown in Table 1. Test design, data processing, and statistical analysis were performed using the Minitab v15.1 software. The Voxler 4 software was used for drawing to reflect the relationship between the variable and the response. The Box-Behnken center composite design was used for the analysis.

\section{Results and Discussion}

3.1. Combined System Analysis. The inlet and outlet air parameters of evaporator were simulated as shown in Figures 5 and 6. They show that the temperature of the evaporator inlet air (return air of the drying box) increases gradually, while the relative humidity, moisture content, and enthalpy decrease gradually. The changes can be mainly attributed to the fact that the water content of the agricultural products and the moisture of the return air decrease gradually as the drying process progresses; thus, the dehumidification of the return air is reduced. Since drying is an approximately isenthalpic process, the return air temperature increases, while the return air relative humidity decreases. During the entire closed drying process, the overall trend of the moisture content of the agricultural products is decreasing, the air inlet temperature of the drying box is fixed, and the overall air inlet enthalpy also decreases; thus, the air outlet enthalpy of the drying box decreases as well. The outlet air enthalpy and relative humidity decrease gradually. The outlet air temperature decreases gradually; however, when the dehumidification capacity is zero, it increases slightly.

The main reason may be that, due to the increase of the inlet air temperature of the evaporator, the unit refrigeration capacity of the refrigerant increases and the inlet air enthalpy exhibits a downward trend; thus, the outlet air enthalpy decreases gradually as well. The reason for that decrease in the temperature should be that, although the inlet air temperature has been increased, the unit cooling capacity has been increased by a greater extent; consequently, the overall outlet air temperature is decreased. As for the slight increase, it may have occurred due to the fact that the unit cooling capacity increases with temperature. After a certain point, the increase is smaller than the increase in temperature; thus, the temperature will increase.

3.2. Response Surface Model and Significance Test. The Minitab 15.1 data analysis software was used to conduct multiple regression fitting analysis on the data in Table 2, and the results are shown in Table 3. Moreover, Voxler 4 was used to draw the relationship between each investigation factor and each test factor (Figure 7).

Table 3 shows the variance analysis of the regression models, where it can be observed that the color difference $Y_{1}$, rehydration ratio $Y_{2}$, and SMER $Y_{3}$ were highly significant $(P<0.001)$. The mismatch items of the $Y_{1}, Y_{2}$, and $Y_{3}$ models were not significant $(P>0.05)$. The results demonstrated that the fitting degree between the regression model and the actual situation was better. The $R^{2}$ values of the $Y_{1}, Y_{2}$, and $Y_{3}$ models were $99.16 \%, 98.95 \%$, and $99.47 \%$, respectively, which were higher than $98 \%$. This indicates that each model can explain more than $98 \%$ of the response value changes, while only less than $2 \%$ of the total variation cannot be explained by the model. In addition, there was a high correlation between the predicted and the actual values, and the test error was small. Therefore, the model can be utilized to analyze and predict the drying indices of Lentinus edodes.

It can be seen in Table 3 that the three regression items of $X_{1}, X_{3}$, and $X_{1}^{2}$ of the $Y_{1}$ model had a significant impact $(P<0.001)$ and the three regression items of $X_{2}, X_{2}^{2}$, and $X_{2} X_{3}$ had also a significant impact $(P<0.05)$, while the other regression items had no significant impact. The $X_{2}$ regression items of the $Y_{2}$ model had a significant impact $(P<0.001)$, and $X_{3}, X_{1}^{2}, X_{2}^{2}$, and $X_{2} X_{3}$ had also a significant impact $(P<0.05)$, while the other regression items had no significant impact $(P>0.05)$. The six regression items of $X_{1}$, $X_{2}, X_{1}^{2}, X_{2}^{2}, X_{3}^{2}$, and $X_{2} X_{3}$ of the $Y_{3}$ model had a significant impact $(P<0.001)$, and the $X_{3}$ and $X_{1} X_{3}$ items had a significant impact as well $(P<0.05)$, while the effects of the others were not significant. The significant items of the above model were kept, the nonsignificant items were eliminated, and a quadratic polynomial regression model of color difference $Y_{1}$, water recovery ratio $Y_{2}$, and specific moisture extraction rate $Y_{3}$ for the three independent variables $\left(X_{1}\right.$, $X_{2}, X_{3}$ ) was developed. Variance analysis was performed for the three regression models, and the three models were fitted on the basis of ensuring that the model is highly significant and the mismatch items are not significant. The regression model can be optimized as equations (12)-(14), where the $x$ value is the code value.

$$
\begin{aligned}
Y_{1}= & 43.902+0.647 X_{1}-2.106 X_{2}+1.213 X_{3}+0.635 X_{1}^{2} \\
& +0.203 X_{2}^{2}-0.850 X_{2} X_{3},
\end{aligned}
$$

$Y_{2}=5.726-0.203 X_{2}+0.075 X_{1}^{2}-0.088 X_{2}^{2}-0.040 X_{2} X_{3}$,

$$
\begin{aligned}
Y_{3}= & 1.630-0.049 X_{1}-0.061 X_{2}-0.016 X_{3}-0.084 X_{1}^{2} \\
& -0.078 X_{2}^{2}-0.093 X_{3}^{2}+0.035 X_{1} X_{3}+0.048 X_{2} X_{3} .
\end{aligned}
$$

3.3. Factor Response Analysis. According to the t-values in Table 3, the influence order of the regeneration temperature $X_{1}$, drying temperature $X_{2}$, and conversion point relative humidity $X_{3}$ on the three factors of the color difference $Y_{1}$ is $X_{2}>X_{3}>X_{1}$. The effect of $X_{1}, X_{2}$, and $X_{3}$ on $Y_{1}$ is shown in Figure $7(\mathrm{a})$. The higher the relative humidity of the conversion point, the greater the color difference value $L$, and vice versa. Furthermore, the higher the relative humidity of the conversion point is, the earlier the conversion valve is closed for closed drying. Thus, the oxygen content in the drying medium is reduced, inhibiting Maillard reaction and increasing the value of $L$. 
TABLE 1: Factors and levels of the response surface test.

\begin{tabular}{lccc}
\hline Level & \multicolumn{2}{c}{ Factors } & \\
& Regeneration temperature $X_{1}\left({ }^{\circ} \mathrm{C}\right)$ & Dry temperature $X_{2}\left({ }^{\circ} \mathrm{C}\right)$ & Conversion point relative humidity $X_{3}(\%)$ \\
\hline-1 & 85 & 50 & 40 \\
0 & 95 & 55 & 45 \\
1 & 105 & 60 & 50 \\
\hline
\end{tabular}

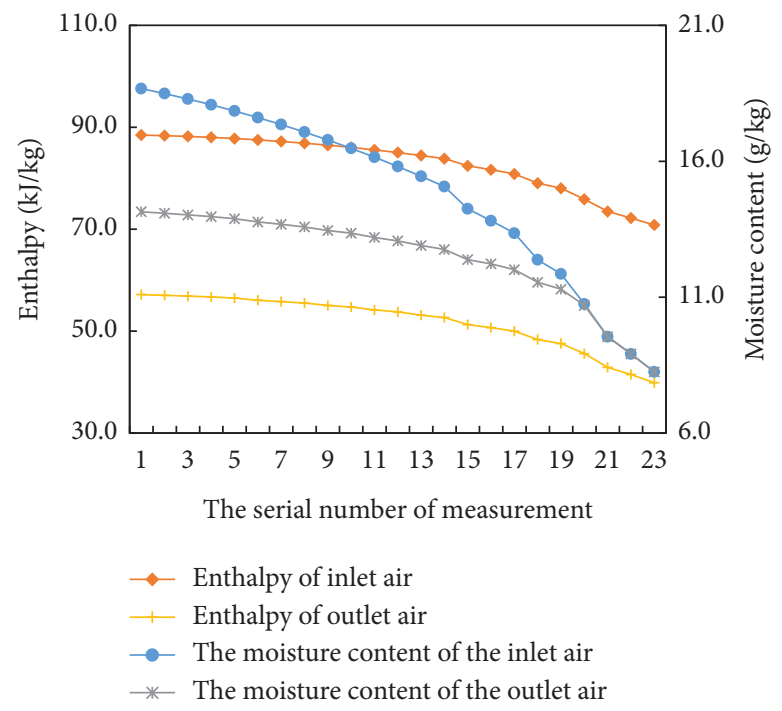

FIGURE 5: Enthalpy and moisture content of the inlet and outlet air of the evaporator.

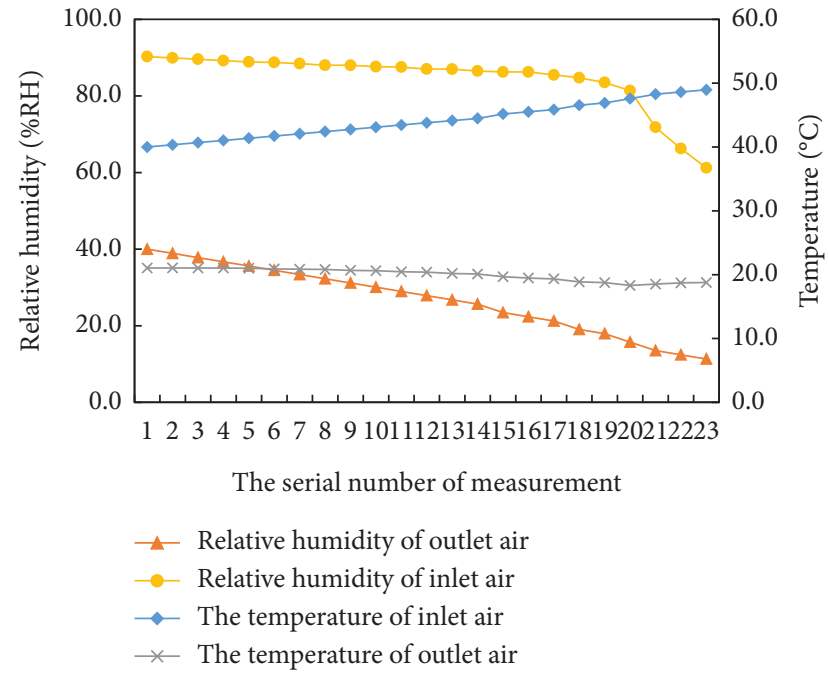

Figure 6: Temperature and relative humidity of the inlet and outlet air of the evaporator.

The influence order of the regeneration temperature $X_{1}$, drying temperature $X_{2}$, and conversion point relative humidity $X_{3}$ on the three factors of the rehydration rate $Y_{2}$ is $X_{2}>X_{3}>X_{1}$. The effect of $X_{1}, X_{2}$, and $X_{3}$ on $Y_{2}$ is shown in Figure $7(\mathrm{~b})$. Overall, the rehydration rate increases with the increase of the conversion point relative humidity, which is similar to its effect on the color difference. In general, the higher the relative humidity of the change point is, the earlier the return air can be used. The related reaction can be restrained, as well as the damage to the pores; thus, the rehydration property is good.

The influence order of the regeneration temperature $X_{1}$, drying temperature $X_{2}$, and conversion point relative humidity $X_{3}$ on the three factors of the unit energy consumption dehumidification capacity $Y_{3}$ is $X_{2}>X_{1}>X_{3}$. The effect of $X_{1}, X_{2}$, and $X_{3}$ on $Y_{3}$ is shown in Figure 7 (c). Overall, the effect of the conversion point relative humidity on SMER is first weak and then strong. The energy-saving effect can be achieved using fresh air before the critical point and return air after it.

3.4. Test Optimization and Verification. To achieve the ideal drying effect, the rehydration ratio of the dried Lentinus edodes should be as high as possible, while the color difference and the SMER should be as low as possible. In order to obtain a high rehydration ratio, the regeneration temperature and drying temperature should be as low as possible. To obtain a mushroom with low color difference, the drying temperature needs to be as low as possible, while the relative humidity at the conversion point should be as high as possible. In order to obtain a dehumidification amount of low energy consumption, the regeneration temperature, drying temperature, and relative humidity at the conversion point should be moderate. 




(a)

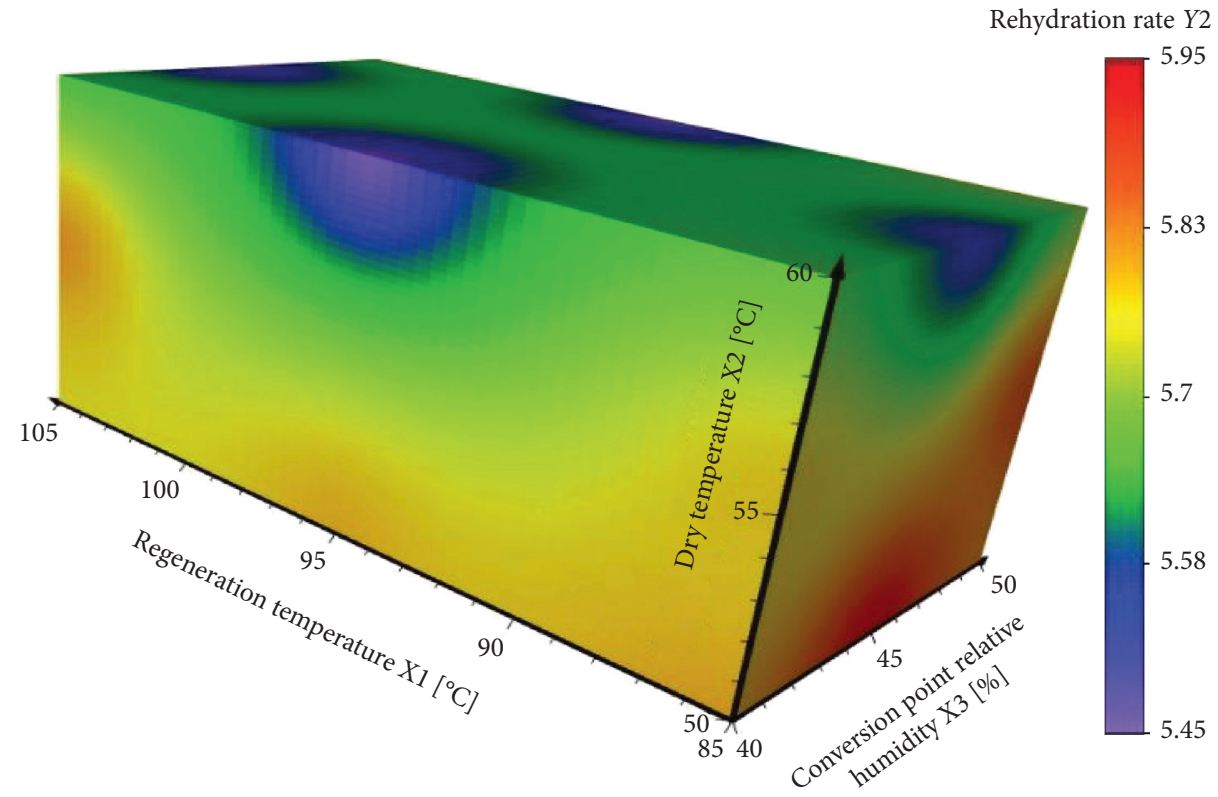

(b)

Figure 7: Continued. 


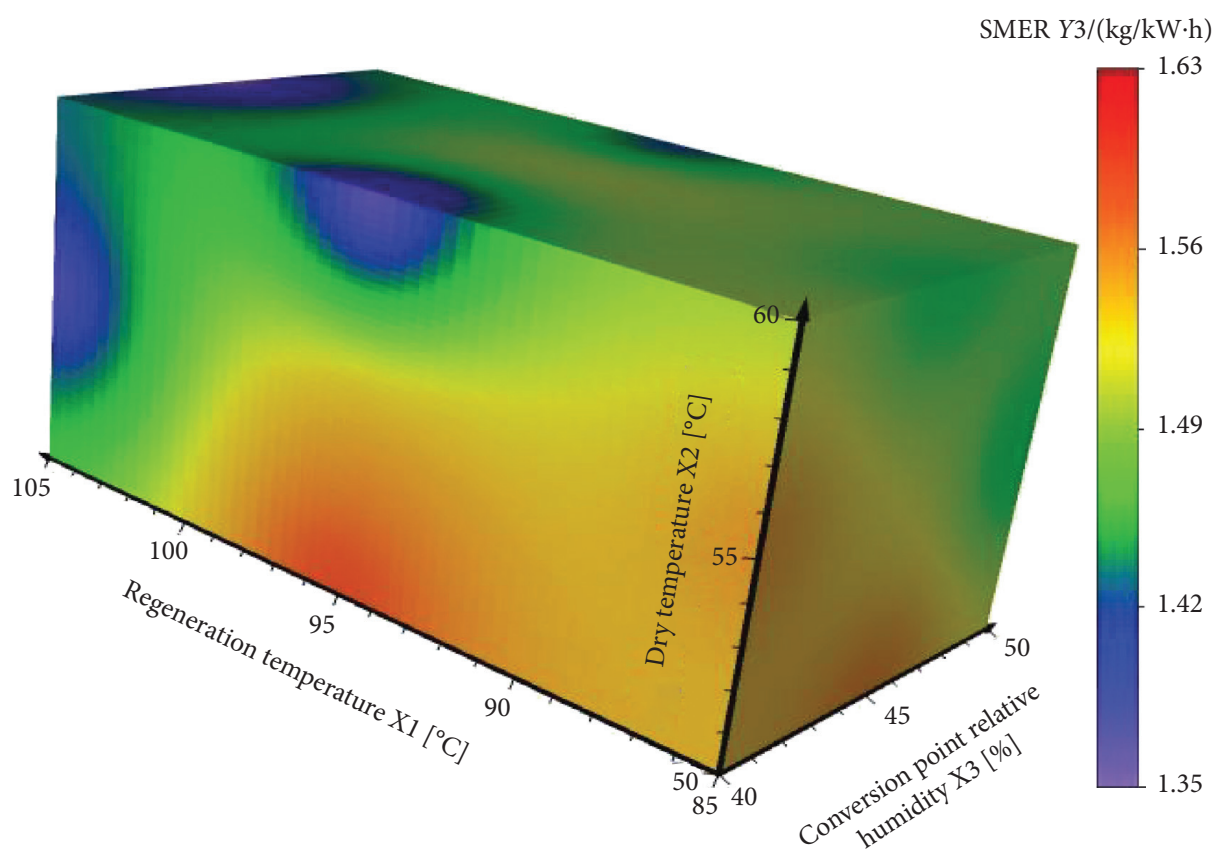

(c)

FIGURE 7: 4D rendering of the relationship between regeneration temperature, drying temperature, conversion point relative humidity, rehydration, color difference, and specific power consumption. (a) Relationship between regeneration temperature, drying temperature, conversion point relative humidity, and color difference. (b) Relationship between regeneration temperature, drying temperature, conversion point relative humidity, and rehydration. (c) Relationship between regeneration temperature, drying temperature, conversion point relative humidity, and specific power consumption.

TABle 2: Experiment design and response values.

\begin{tabular}{|c|c|c|c|c|c|c|}
\hline No. & $\begin{array}{c}\text { Regeneration } \\
\text { temperature X1 }\left({ }^{\circ} \mathrm{C}\right)\end{array}$ & $\begin{array}{c}\text { Dry temperature } \\
\mathrm{X} 2\left({ }^{\circ} \mathrm{C}\right)\end{array}$ & $\begin{array}{c}\text { Conversion point relative } \\
\text { humidity X3 }(\%)\end{array}$ & $\begin{array}{c}\text { Color } \\
\text { difference Y1 }\end{array}$ & $\begin{array}{l}\text { Rehydration ratio } \\
\text { Y2 }\end{array}$ & $\begin{array}{c}\text { SMERY3 [kg/ } \\
\mathrm{kW} \bullet \mathrm{h}]\end{array}$ \\
\hline 1 & 1 & 0 & -1 & 44.1 & 5.81 & 1.38 \\
\hline 2 & 0 & -1 & -1 & 44.4 & 5.79 & 1.59 \\
\hline 3 & -1 & 1 & 0 & 42.3 & 5.55 & 1.47 \\
\hline 4 & 0 & 1 & -1 & 41.7 & 5.46 & 1.37 \\
\hline 5 & 1 & 0 & 1 & 46.3 & 5.8 & 1.43 \\
\hline 6 & 0 & 1 & 1 & 42.4 & 5.45 & 1.42 \\
\hline 7 & 0 & 0 & 0 & 43.9 & 5.74 & 1.62 \\
\hline 8 & 0 & 0 & 0 & 43.8 & 5.73 & 1.64 \\
\hline 9 & -1 & 0 & -1 & 42.9 & 5.77 & 1.55 \\
\hline 10 & 0 & -1 & 1 & 48.5 & 5.94 & 1.45 \\
\hline 11 & -1 & 0 & 1 & 45.6 & 5.87 & 1.46 \\
\hline 12 & -1 & -1 & 0 & 45.5 & 5.95 & 1.56 \\
\hline 13 & 1 & 0 & 0 & 45.2 & 5.78 & 1.49 \\
\hline 14 & 0 & -1 & 0 & 46.4 & 5.8 & 1.62 \\
\hline 15 & 1 & 1 & 0 & 43.2 & 5.46 & 1.35 \\
\hline
\end{tabular}

When the regeneration temperature is $95.5^{\circ} \mathrm{C}$, the drying temperature is $52.5^{\circ} \mathrm{C}$, and the relative humidity of the conversion point is $45.6 \%$, the color difference is 44.9 , the rehydration ratio is 5.8 , and the $S M E R$ is $1.64 \mathrm{~kg} / \mathrm{kW} \cdot \mathrm{h}$. The critical dehumidification method (Section 2.1.2) determined that the optimal conversion point humidity is about $46 \%$, which was in accord with the experimental results. To validate the optimization results, the optimized parameter combination was tested and verified. When the regeneration temperature was $96^{\circ} \mathrm{C}$, the drying temperature was $53^{\circ} \mathrm{C}$, and the relative humidity of the conversion point was $46 \%$; the average value was obtained by performing the same test 3 times. As it can be seen in Figure 8, the dried Lentinus edodes exhibited a small shrinkage and little color change. The color difference of the dried Lentinus edodes is 46.3 , the rehydration ratio is 5.75 , the $S M E R$ is $1.62 \mathrm{~kg} / \mathrm{kW} \bullet \mathrm{h}$, and the 
TABLE 3: Variance analysis of response surface model.

\begin{tabular}{|c|c|c|c|c|c|c|c|c|c|c|c|c|}
\hline \multirow{2}{*}{ Item } & \multicolumn{4}{|c|}{ Color difference e $Y_{1}$} & \multicolumn{4}{|c|}{ Rehydration ratio $Y_{2}$} & \multicolumn{4}{|c|}{ SMER $Y_{3}$} \\
\hline & Df & $T$ & $P$ & Coefficient & Df & $T$ & $P$ & Coefficient & Df & $T$ & $P$ & Coefficient \\
\hline Constant & 1 & 234.686 & $* * *$ & 43.902 & 1 & 301.544 & $* * *$ & 5.725 & 1 & 208.704 & $* * *$ & 1.630 \\
\hline$X_{1}$ & 1 & 5.970 & 0.002 & 0.647 & 1 & -1.860 & 0.122 & -0.020 & 1 & -10.858 & $* * *$ & -0.049 \\
\hline$X_{2}$ & 1 & -19.442 & $* * *$ & -2.106 & 1 & -18.484 & $* * *$ & -0.203 & 1 & -13.571 & $* * *$ & -0.061 \\
\hline$X_{3}$ & 1 & 12.126 & $* * *$ & 1.213 & 1 & 2.833 & 0.037 & 0.029 & 1 & -3.893 & 0.011 & -0.016 \\
\hline$X_{1}^{2}$ & 1 & 3.804 & 0.013 & 0.635 & 1 & 4.399 & 0.007 & 0.075 & 1 & -11.999 & $* * *$ & -0.084 \\
\hline$X_{2}^{\frac{1}{2}}$ & 1 & 1.217 & 0.278 & 0.203 & 1 & -5.206 & 0.003 & -0.088 & 1 & -11.151 & $* * *$ & -0.078 \\
\hline$X_{3}^{3}$ & 1 & 1.120 & 0.314 & 0.167 & 1 & 1.186 & 0.289 & 0.018 & 1 & -14.946 & $* * *$ & -0.093 \\
\hline$X_{1} X_{2}$ & 1 & -1.946 & 0.109 & -0.364 & 1 & -0.601 & 0.574 & -0.011 & 1 & -1.741 & 0.142 & -0.014 \\
\hline$X_{1} X_{3}$ & 1 & -0.884 & 0.417 & -0.125 & 1 & -1.916 & 0.113 & -0.028 & 1 & 5.929 & 0.002 & 0.035 \\
\hline$X_{2} X_{3}$ & 1 & -6.011 & 0.002 & -0.850 & 1 & -2.787 & 0.039 & -0.040 & 1 & 8.046 & $* * *$ & 0.048 \\
\hline Regression & 9 & 65.84 & $* * *$ & & 9 & 52.38 & $* * *$ & & 9 & 104.77 & $* * *$ & \\
\hline Linear & 3 & 175.90 & $* * *$ & & 3 & 129.29 & $* * *$ & & 3 & 134.42 & $* * *$ & \\
\hline Square & 3 & 5.14 & 0.055 & & 3 & 26.76 & 0.002 & & 3 & 128.35 & $* * *$ & \\
\hline Interaction & 3 & 13.57 & 0.008 & & 3 & 3.93 & 0.087 & & 3 & 34.31 & 0.001 & \\
\hline Lack of fit & 3 & 19.75 & 0.167 & & 3 & 20.35 & 0.165 & & 3 & 0.62 & 0.727 & \\
\hline Total & \multicolumn{4}{|c|}{$R-\mathrm{sq}=99.16 \% \mathrm{R}-\mathrm{Sq}(\mathrm{adj})=97.66 \%$} & \multicolumn{4}{|c|}{$\mathrm{R}-\mathrm{sq}=98.95 \% \mathrm{R}-\mathrm{Sq}(\mathrm{adj})=97.06 \%$} & \multicolumn{4}{|c|}{$R-\mathrm{sq}=99.47 \% ; R-\mathrm{Sq}($ adj $)=98.52 \%$} \\
\hline
\end{tabular}

Note. $* * *$ Significance $(P<0.001)$.

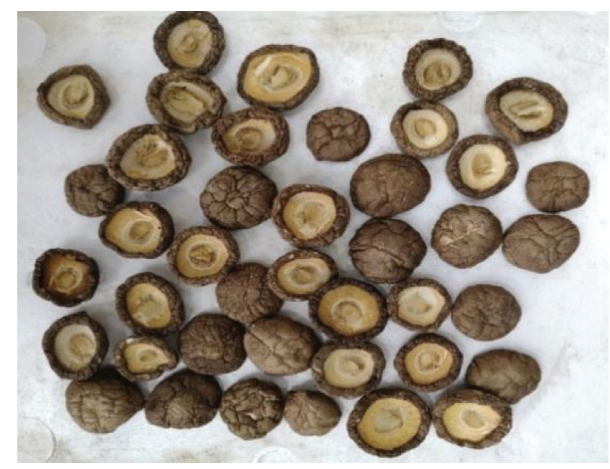

Figure 8: Dried Lentinus edodes.

quality is better than that obtained under heat pump drying [30]. The test results were close to the predicted values, which verifies the reliability of the model.

\section{Conclusions}

The factors that affect the drying quality and the energy consumption of the system combining heat pump drying and desiccant wheel dehumidification include the regeneration temperature, drying temperature, and conversion point relative humidity. The effect of the conversion point relative humidity on SMER appeared weak first and then strong.

The optimal conversion point humidity was about $46 \%$ $\mathrm{RH}$, which was slightly different from the test optimization value of $45.6 \% \mathrm{RH}$. In addition, the $S M E R$ was found to be $1.62 \mathrm{~kJ} / \mathrm{kg}$, indicating that the proposed method has low energy consumption and meets the requirements of agricultural drying.

The results demonstrated that the color difference of the Lentinus edodes was 46.3 and the rehydration ratio was 5.75. The drying quality was better than that of heat pump drying, which also meets the drying requirements for agricultural products.

\section{Data Availability}

The data used to support the findings of this study are included within the article.

\section{Conflicts of Interest}

The authors declare that they have no conflicts of interest.

\section{Authors' Contributions}

All authors contributed equally to the present study.

\section{Acknowledgments}

This research was supported by a Special Fund for Basic Scientific Research and Business Expenses of Central Public Welfare Scientific Research Institutes (S202006-02, S20200603); Independent Innovation Fund of Agricultural Science and Technology in Jiangsu Province (CX (19) 3117); Agricultural Science and Technology Innovation Program of the Chinese Academy of Agricultural Sciences-Drying and Processing Equipment Team of Special Agricultural Products (2021); and the National Key Research and Development Program of China (2020YFD1000300).

\section{References}

[1] M. J. Pikal, R. Bogner, V. Mudhivarthi, P. Sharma, and P. Sane, "Freeze-drying process development and scale-up: scale-up of edge vial versus center vial heat transfer coefficients, K v," Journal of Pharmaceutical Sciences, vol. 105, no. 11, pp. 3333-3343, 2016.

[2] G. P. Li, H. X. Xie, J. L. Wang, J. C. Yan, H. Wei, and Z. K. Chen, "Design and experiment of online dual-mode measuring device for moisture content in hot air drying process of fruits and vegetables based on mcu," Journal of Agricultural Mechanization Research, vol. 41, no. 09, pp. 199-206, 2019. 
[3] I. Boutelba, S. Zid, P. Glouannec, A. Magueresse, and S. Youcef-ali, "Experimental data on convective drying of potato samples with different thickness," Data in Brief, vol. 18, pp. 1567-1575, 2018.

[4] M. K. M. Ali, A. Fudholi, M. S. Muthuvalu, J. Sulaiman, and S. M. Yasir, "Implications of drying temperature and humidity on the drying kinetics of seaweed," in AIP Conference Proceedingsvol. 1905, no. 1, AIP Publishing LLC, Article ID 050004, 2017.

[5] B. Önal, G. Adiletta, A. Crescitelli, M. Di Matteo, and P. Russo, "Optimization of hot air drying temperature combined with pre-treatment to improve physico-chemical and nutritional quality of "Annurca" apple," Food and Bioproducts Processing, vol. 115, pp. 87-99, 2019.

[6] H. Y. Ju, S. H. Zhao, H. Y. Zhao, Z. A. Zheng, Z. J. Gao, and H. W. Xiao, "Effect of relative humidity on drying characteristic and quality of panacis quinquefolii radix," Chinese Traditional and Herbal Drugs, vol. 51, no. 3, pp. 631-638, 2020.

[7] J. A. Figueiredo, A. M. Lago, J. M. Mar, L. S. Silva, E. A. Sanches, and T. P. Souza, "Stability of camu-camu encapsulated with different prebiotic biopolymers," Journal of the Ence of Food and Agriculture, vol. 100, no. 8, pp. 34713480, 2020.

[8] D. K. Tagantsev and D. V. Ivanenko, "Validity of the second Fick's law for modeling ion-exchange diffusion in noncrystalline viscoelastic media (glasses)," Journal of Applied Physics, vol. 119, no. 13, Article ID 134905, 2016.

[9] P. Wang, Y. Chen, S. G. Li, C. F. Li, and B. Q. Yang, "Dried characteristics and quality of different parts of jujube (junzao) under temperature and humidity variable drying methods in the southern of xinjiang," Food Science \& Technology, vol. 41, no. 12, pp. 14-19, 2016.

[10] R. Tu, X.-H. Liu, and Y. Jiang, "Lowering the regeneration temperature of a rotary wheel dehumidification system using exergy analysis," Energy Conversion and Management, vol. 89, pp. 162-174, 2015.

[11] A. Alishah, M. V. Kiamahalleh, F. Yousefi, A. Emami, and M. V. Kiamahalleh, "Solar-assisted heat pump drying of coriander: an experimental investigation," International Journal of Air-Conditioning and Refrigeration, vol. 26, no. 4, pp. 1850037.1-1850037.13, 2018.

[12] Q. R. Wu, G. M. Wang, E. Zeng et al., "Advances on heat pump combined drying of agricultural products," Food Science \& Technology, vol. 41, no. 11, pp. 88-92, 2016.

[13] M. M. S. Dezfouli, S. Mat, G. Pirasteh, K. S. M. Sahari, K. Sopian, and M. H. Ruslan, "Simulation analysis of the four configurations of solar desiccant cooling system using evaporative cooling in tropical weather in Malaysia," International Journal of Photoenergy, vol. 2014, no. 11, pp. 1-14, 2014.

[14] X. D. Liang, Research on Inteligent Control System for Air Source Heat Pump Machine and Drying Room Based on Climatix Platform, Beijing university of technology, Beijing, China, 2018.

[15] A. Taşcıkaraoğlu, N. G. Paterakis, O. Erdinç, and J. P. Catalão, "Combining the flexibility from shared energy storage systems and DLC-based demand response of HVAC units for distribution system operation enhancement," IEEE Transactions on Sustainable Energy, vol. 10, no. 1, pp. 137-148, 2018.

[16] X. H. Bai and B. G. Li, "Study on drying characteristics and mathematical model for heat pump drying of mushroom," Journal of Refrigeration, vol. 39, no. 04, pp. 42-48, 2018.

[17] M. Jokiel, M. Bantle, C. Kopp, and E. H. Verpe, "Modelicabased modelling of heat pump-assisted apple drying for varied drying temperatures and bypass ratios," Thermal Science and Engineering Progress, vol. 19, Article ID 100575, 2020.

[18] S. C. Liu, X. Q. Liu, M. J. Sun, H. L. Liu, and Z. L. Sun, "Experimental investigation on drying performance of an existed enclosed fixed frequency air source heat pump drying system," Applied Thermal Engineering, vol. 130, pp. 735-744, 2018.

[19] L. Taeri, M. Akta, S. Evik, M. Gülcü, G. U. Sekin, and B. Aktekeli, "Determination of drying kinetics and quality parameters of grape pomace dried with a heat pump dryer," Food Chemistry, vol. 260, pp. 152-159, 2018.

[20] J. Lü, J. Wei, Z. T. Zhang, L. W. Yang, Z. Li, and Z. M. Li, "Theoretical analysis and comparison of performance on heat pump system for flue-cured tobacco based on isenthalpic and isothermal process," Transactions of the Chinese Society of Agricultural Engineering, vol. 28, no. 20, pp. 265-271, 2012.

[21] J. H. Li, "Research on heat pump drying equipment for practical heat-sensitive materials," Journal of Chinese Agricultural Mechanization, vol. 39, no. 02, pp. 46-48, 2018.

[22] H. K. Zhang, Study on the Thermodynamics of Heat Pump Drying System and the Drying Process of Typical Materials, Doctoral dissertation, China Agricultural University, Beijing, China, 2005.

[23] B. G. Zhang, "Critical dehumidified state during dehumidification drying," Journal of Beijing Forestry University, vol. 29, no. 6, pp. 181-184, 2007.

[24] J. L. Wang, W. D. Song, C. Q. Jing et al., "Structural design and process parameter optimization of heat pump drying system of wheel dehumidification for pleurotus eryngii," Transactions of the Chinese Society of Agricultural Engineering, vol. 35, no. 04, pp. 273-280, 2019.

[25] Z. H. Miao, Research on Heat-Moisture Process and Performance of Closed Heat Pump Drying Dishwasher, Master dissertation, South China University of Technology, Guangzhou, China, 2020.

[26] J. Teng and Y. Chen, "Comparison and analysis of variable speed compressor models," Refrigeration, vol. 47, no. 2, pp. 79-90, 2019.

[27] W. Kim and J. E. Braun, "Virtual refrigerant mass flow and power sensors for variable-speed compressors," in Proceedings of the West Lafayette: International refrigeration and Air conditioning conference, pp. 1-8, West Lafayette, IN, USA, July 2012.

[28] J. L. Wang, W. D. Song, T. H. Ding et al., "Wheel dehumidification heat pump drying system and dehumidification method," Applied Thermal Engineering, vol. 148, pp. 78-86, 2019.

[29] J. L. Wang, W. D. Song, M. Y. Wang, T. H. Ding, W. Jinji, and S. Cheng, "Structural design and dry parameter optimization of far infrared dryer for hericium erinaceus mycelium," International Agricultural Engineering Journal, vol. 28, no. 3, pp. 57-63, 2019.

[30] J. L. Wang, C. Q. Jin, W. D. Song, T. H. Ding, M. Y. Wang, and J. J. Wu, "Design and test of drying system for desiccant wheel dehumidification," Transactions of the Chinese Society for Agricultural Machinery, vol. 51, no. 11, pp. 374-383, 2019. 